\title{
High pressure study of a Zr-based bulk metallic glass and its composite
}

\author{
Itzhak Halevy I, II, Ersan Üstündag*, I, S. Salhov ${ }^{\text {II }}$, Alan F. Yue ${ }^{\text {I }, ~ A . ~ B r o i d e ~}{ }^{\text {II }}$ and Jingzhu Hu ${ }^{\text {III }}$ \\ I Department of Materials Science, California Institute of Technology, Pasadena, CA 91125, USA \\ II Nuclear Research Center - Negev, P.O. Box 9001, Beer-Sheva, Israel \\ ${ }^{\text {III }}$ Geophysical Laboratory of Carnegie Institution of Washington, Washington, DC 20015, USA
}

Received January 16, 2004; accepted January 26, 2004

\section{Metallic glass / Pressure / Elastic strain / PDF analysis / Zr-based bulk metallic glass}

\begin{abstract}
Energy dispersive X-ray diffraction was used to study the structural evolution of a bulk metallic glass (BMG) alloy and its composite with $\mathrm{W}$ particles under hydrostatic pressure. The diffraction data, especially the pair distribution function (PDF) of the BMG allowed the direct measurement of its elastic strain. The results suggest that PDF patterns of BMGs can be used to deduce their strain evolution in composites as well as in monolithic form. Although the PDF method of strain measurement in amorphous alloys offers lower resolution compared to the analysis of Bragg reflections from crystalline materials, the PDF techique yields valuable information about the deformation of BMGs.
\end{abstract}

\section{Introduction}

Bulk metallic glasses (BMGs) are recently developed amorphous alloys with extremely low critical cooling rates that permit the casting of large specimens with diameters greater than $50 \mathrm{~mm}$ [1]. The mechanical properties of BMGs are impressive and make these materials attractive for structural applications. The yield strength of most BMGs is above 2 $\mathrm{GPa}$, their elastic strain limit is about $2 \%$, their fracture toughness is above $20 \mathrm{MPa} \cdot \mathrm{m}^{1 / 2}$, and they possess a specific strength that is higher than that of most steels.

The only major drawback BMGs suffer at ambient temperatures is their lack of damage tolerance and ductility. Under unconstrained loading (e.g., uniaxial tension or compression) BMGs fail by forming highly localized shear bands and experience negligible plastic deformation. To overcome this problem, various BMG matrix composites have been developed in recent years [2-4]. The ductile reinforcements used in these composites appear to prevent shear localization and promote the formation of multiple shear bands which in turn increase the ductility

\footnotetext{
* Correspondence author (e-mail: ersan@caltech.edu)
}

of the BMG by delaying its fracture. As a result, BMG composites often exhibit more damage tolerance and ductility compared to monolithic BMGs.

The details of the microstructural deformation mechanisms in BMG composites are poorly understood at the moment. This lack of fundamental understanding precludes further optimization of their mechanical properties. Recent work by Üstündag and co-workers [5-8] has employed an integrated experimental-theoretical approach to study the deformation of BMG matrix composites. Using either high energy X-ray diffraction (XRD) [5, 6] or neutron diffraction $[7,8]$, they measured the lattice strain in the crystalline reinforcements and then used mechanics modeling to deduce the behavior of the BMG matrix indirectly. The results provided valuable insight and showed that the reinforcements yield first, and at a later stage of the deformation, induce multiple shear bands in the BMG matrix. The extent of the shear band multiplication strongly depends on reinforcement geometry (namely, the more the BMG is constrained, the higher the shear band density), stiffness mismatch between the two phases as well as the strength of the interfaces between the matrix and reinforcements. Current work at Caltech aims to quantify these effects and attain a better understanding of the mechanisms that control the deformation of BMG composites.

The previous diffraction work on these materials [5-8] employed the crystalline reinforcements as "internal strain gages" to monitor their deformation in situ by tracking shifts of their Bragg reflections as a function of applied stress. The behavior of the amorphous matrix could only be deduced via mechanics modeling. Model comparison and validation would have been more rigorous had it been possible to measure strain in the BMG matrix as well. The high strength of BMGs and their relatively low Young's modulus ( $80-100 \mathrm{GPa})$ offer a unique opportunity to apply high stresses to these materials without yielding or fracturing them while inducing a large elastic strain response. The main objective of the present article is to measure elastic strain in a BMG alloy $\left(\mathrm{Zr}_{57} \mathrm{Nb}_{5} \mathrm{Al}_{10} \mathrm{Cu}_{15.4} \mathrm{Ni}_{12.6}\right)$ in both monolithic and composite form as it deforms under hydrostatic pressure. Such a loading geometry was chosen to eliminate the possibility of plastic deformation or failure while imposing well de- 
fined boundary conditions. Preliminary results of a simple analysis are presented below. As far as the authors are aware, this is the first time diffraction has been used to extract elastic strain data from an amorphous metal. A relevant study is noted here by Egami and co-workers [9] who, too, employed energy dispersive XRD to investigate creep-induced structural anisotropy in a metallic glass and demonstrated the power of PDF analysis in studying amorphous materials.

\section{Experimental procedure}

The BMG alloy used in this study $\left(\mathrm{Zr}_{57} \mathrm{Nb}_{5} \mathrm{Al}_{10} \mathrm{Cu}_{15.4} \mathrm{Ni}_{12.6}\right.$ or Vitreloy $106^{\mathrm{TM}}$ ) is one of the best $\mathrm{Zr}$-based glass forming alloys. Both a monolithic Vit. 106 and its composite with 20 vol. $\% \mathrm{~W}$ particles were studied. The particulate composites of Vit. 106 were investigated before $[5,6]$ using high energy XRD. These studies showed that the particulate composites are good model systems to investigate the deformation of BMG composites and are especially suitable for analysis using the Eshelby model [10] which was also utilized in the present study.

Figure 1 shows a typical micrograph of the W-BMG composite. Electron microscopy and XRD investigations confirmed the amorphous structure of the BMG in both the monolith and as the matrix of the composite. In the latter sample, digital image processing revealed that the average $\mathrm{W}$ particle size was about $10 \mu \mathrm{m}$. Electron microprobe analysis confirmed the chemical composition of all phases and the lack of interdiffusion between the $\mathrm{W}$ and the Vit. 106 matrix during processing.

The structure of the two specimens under ambient conditions was determined with XRD using a sealed tube $\mathrm{Cu} K_{\alpha}$ source $(\lambda=1.54056 \AA)$. Si powder (NIST, Standard Reference Material 640a) was employed as an internal standard to minimize the displacement error [11] and to allow direct comparison with the high pressure data.

To systematically investigate the effect of pressure on the structure of BMG and its composite, energy dispersive

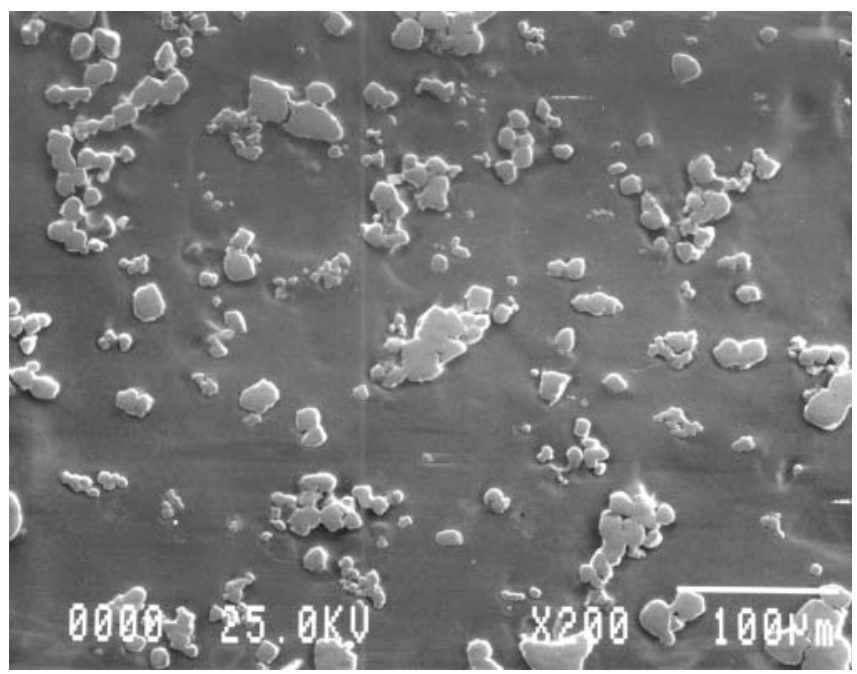

Fig. 1. SEM backscattered electron image of Vitreloy 106 $\left(\mathrm{Zr}_{57} \mathrm{Nb}_{5} \mathrm{Al}_{10} \mathrm{Cu}_{15.4} \mathrm{Ni}_{12.6}\right)-20 \% \mathrm{~W}$ composite. The $\mathrm{W}$ particles (light colored) are on average about $10 \mu \mathrm{m}$ in size.
X-ray diffraction studies were performed at the X17 beamline of the National Synchrotron Light Source (NSLS), Brookhaven National Laboratory using a $50 \mu \mathrm{m}$ diameter spot size. Pressure (up to $31.5 \mathrm{GPa}$ ) was applied via a Merrill-Bassett type diamond anvil cell (DAC) [12] and was measured by the fluorescence of ruby technique [13]. Energy dispersive data were collected with a Ge detector at a fixed Bragg angle $\left(2 \theta=13^{\circ}\right)$. The pressure distribution inside the sampling volume was checked in different regions and was determined to vary by less than $5 \%$. Typical data collection time was around 20 minutes at each pressure value. The sample dimensions were about $50 \mu \mathrm{m}$ in height and $150 \mu \mathrm{m}$ in diameter while the DAC culets were $500 \mu \mathrm{m}$ in diameter. $\mathrm{Si}$ oil was used as the pressure medium in the $250 \mu \mathrm{m}$ diameter cavity of a stainless steel gasket.

\section{Data analysis}

\section{X-ray diffraction data}

To analyze the XRD results, first, energy dispersive data from NSLS were converted to angle dispersive data using conventional energy-to-wavelength relationships. The X-ray energy at NSLS ranged from about $3 \mathrm{keV}(\lambda \sim 4 \AA)$ to about $70 \mathrm{keV}(\lambda \sim 0.17 \AA)$ yielding a $Q(=4 \pi \sin (\theta) / \lambda)$ range of 0.5 to $8 \AA^{-1}$. However, the $Q=0.5$ to $2.2 \AA^{-1}$ region contains fluorescence peaks which complicate data interpretation. Therefore, this range has been omitted in Figs. 2 and 3 for the monolithic BMG and the composite, respectively. Figure 2 reveals a prominent amorphous peak at $Q \sim 2.6 \AA^{-1}$, a second one at $\sim 4.3 \AA^{-1}$ and a much weaker one at $6.5 \AA^{-1}$. The first two peaks are also visible in the pattern from the composite (Fig. 3). Another important result exhibited in both figures is the lack of pressureinduced crystallization in BMG. This is in sharp contrast

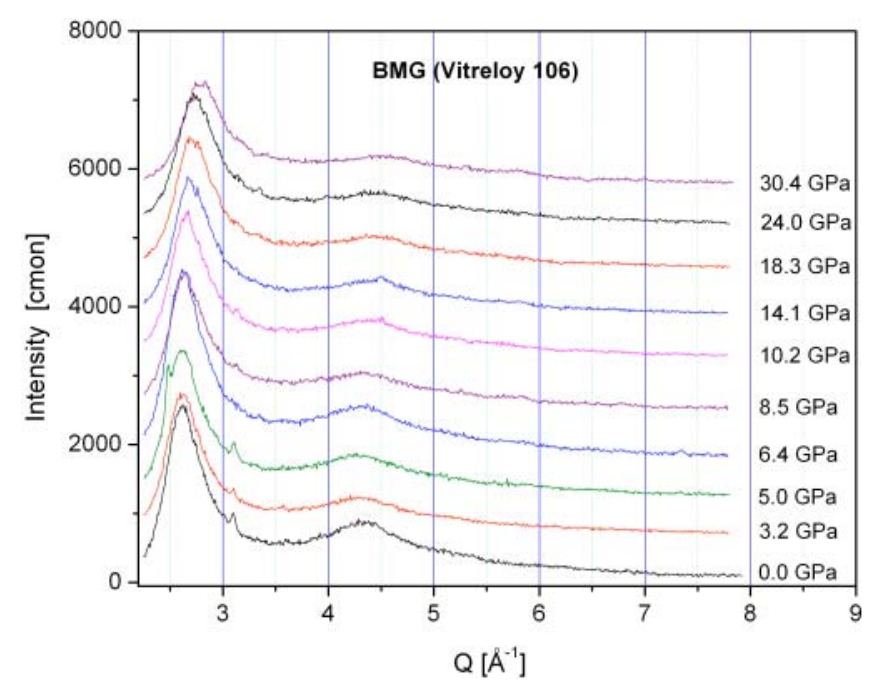

Fig. 2. The amorphous spectra of the monolithic Vit. 106 $\left(\mathrm{Zr}_{57} \mathrm{Nb}_{5} \mathrm{Al}_{10} \mathrm{Cu}_{15.4} \mathrm{Ni}_{12.6}\right)$ as a function of pressure obtained using energy dispersive X-ray diffraction at NSLS beamline X17. A systematic shift towards higher $Q$ values (or lower $d$ spacings) is observed as pressure increases. Also, there is no detectable pressureinduced crystallization or phase transformation. The small peaks seen in some patterns are from the steel gasket. 


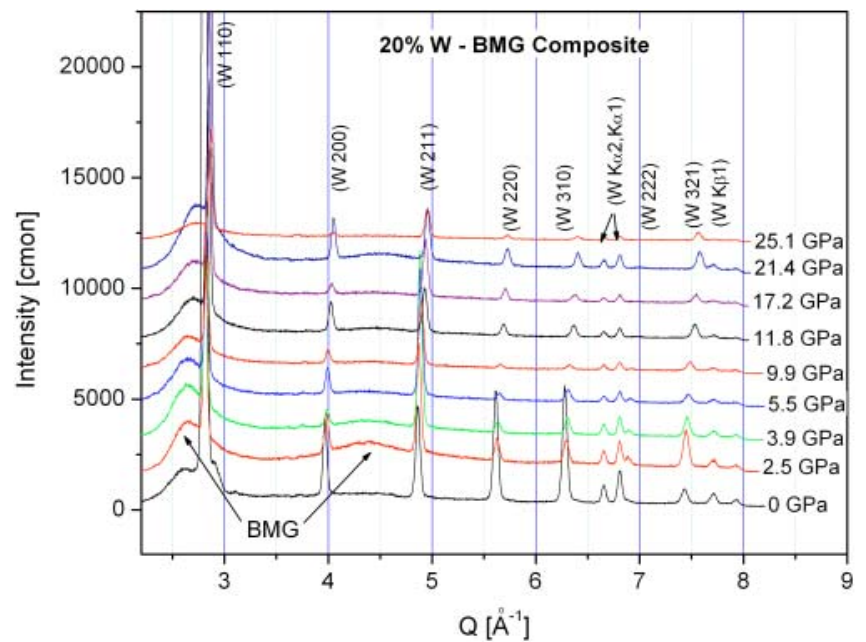

Fig. 3. The diffraction data of the $\mathrm{Zr}_{57} \mathrm{Nb}_{5} \mathrm{Al}_{10} \mathrm{Cu}_{15.4} \mathrm{Ni}_{12.6}$ matrix/ $20 \% \mathrm{~W}$ composite as a function of pressure obtained using energy dispersive X-ray diffraction. Some $\mathrm{W}$ reflections are indexed.

with the observations by $\mathrm{He}$ et al. [14] who studied a slightly different Zr-based BMG alloy and claimed pressure-induced crystallization around $25 \mathrm{GPa}$.

To obtain further insight into the structure of the BMG alloy used in the present investigation, its atomic pair distribution function (PDF) [15] was calculated employing the RAD program [16] using:

$$
G(r)=\frac{2}{\pi} \int_{0}^{\infty} Q[S(Q)-1] \sin (Q r) \mathrm{d} Q .
$$

Here $S(Q)$ is the structure-dependent part of the diffraction data. Note that the $\mathrm{W}$ peaks were subtracted from the diffraction pattern of the composite before calculating BMG's PDF. This was done by, first, fitting the whole composite diffraction pattern using the Rietveld method as described below (the BMG part was fit with a background function). Then the crystalline part of the Rietveld model representing the $\mathrm{W}$ contribution was subtracted. The remaining data (between $Q=2.2$ to $8 \AA^{-1}$ ) were subsequently used in the RAD program to calculate the PDF pattern of the BMG matrix. The results are exhibited in Figs. 4 and 5 for the BMG in the monolith and the WBMG composite, respectively. Both patterns confirm the lack of pressure-induced crystallization in BMG. In addition to the systematic peak shifts toward lower $r$ values as pressure increases, there are also subtle changes in the second PDF peak, especially in the monolith. Unfortunately, the limited $Q$ space available in this study might have led to oscillations in the PDF patterns [15] precluding any further interpretation of the data in Figs. 4 and 5. In addition, atomistic modeling of BMG [17] is underway to fully appreciate these effects and to better quantify the influence of applied pressure on the structure of the alloy.

\section{Deformation analysis}

The PDF patterns of BMG (Figs. 4 and 5) clearly show a systematic shift of the first peak towards lower $r$ values as a function of pressure. To quantify this contraction of the BMG, its first peak in both the PDF and XRD patterns

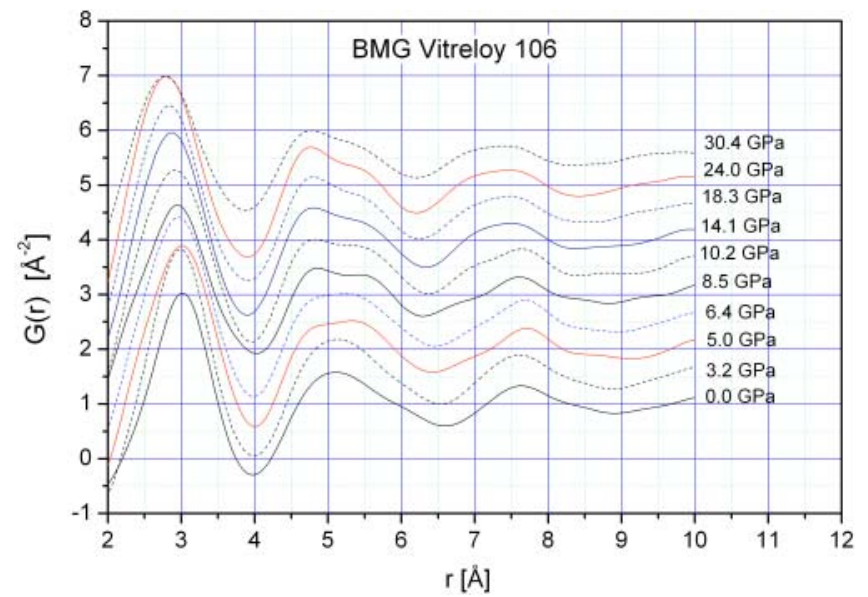

Fig. 4. $\mathrm{PDF}$ of the monolithic $\mathrm{BMG}\left(\mathrm{Zr}_{57} \mathrm{Nb}_{5} \mathrm{Al}_{10} \mathrm{Cu}_{15.4} \mathrm{Ni}_{12.6}\right)$ at various pressures. A systematic shift towards a lower average atomic spacing is observed.

was fit with a Gaussian. At this point, an additional assumption is made that the shift of this peak is representative of the average deformation of BMG under hydrostatic pressure. Since the first peak signifies the average first nearest neighbor distance in the glass structure, this assumption is reasonable, but it needs to be further quantified via molecular dynamics simulations (currently in progress [17]). A similar assumption was made by Meade et al. [18] who studied the deformation of $\mathrm{SiO}_{2}$ glass under hydrostatic pressure. In the absence of pressure-induced structural transformations, they related the linear strain $(\varepsilon)$ due to the elastic compression of the glass to the volume change and peak shift as:

$$
1+\varepsilon=\left[\frac{V}{V_{0}}\right]^{1 / 3}=\frac{d}{d_{0}}=\frac{Q_{0}}{Q} \quad \text { where } \quad d=\frac{2 \pi}{Q} .
$$

Here, $d$ and $Q$ indicate the position of the first peak of BMG and the subscript " 0 " refers to their values at ambient pressure.

The structure of the composite was analyzed by the Rietveld method $[19,20]$. The BCC structure of W (Im3m

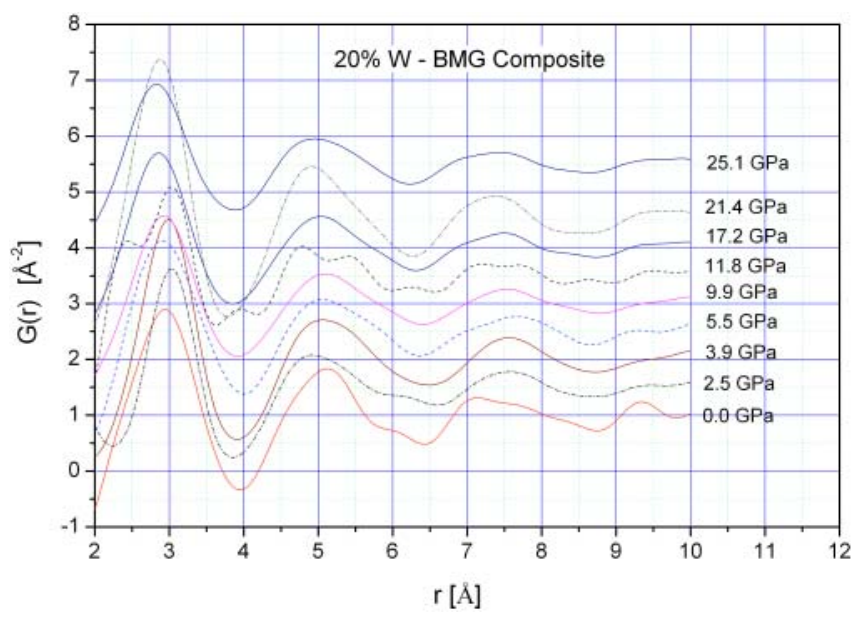

Fig. 5. PDF of the BMG matrix in the W-BMG composite as a function of pressure. The $\mathrm{W}$ peaks were subtracted before the calculation of these PDF patterns. The limited $Q$ range available from the XRD data likely contributed to some of the oscillations seen in these patterns (e.g., the one around $2.2 \AA$ for the $11.8 \mathrm{GPa}$ pattern). 
space group, lattice parameter, $a \cong 3.165 \AA$ ) was confirmed throughout the experiment indicating no pressureinduced phase transformation. The lattice strain in W was calculated from its lattice constant and related to volume change as shown in Eq. (2).

The relationship between pressure and volume change for both phases was determined by the Vinet equation of state [21]:

$$
\begin{aligned}
P(V)= & 3 B_{0}\left(\frac{V}{V_{0}}\right)^{-2 / 3}\left[1-\left(\frac{V}{V_{0}}\right)^{1 / 3}\right] \\
& \times \exp \left[\frac{3}{2}\left(B_{0}^{\prime}-1\right)\left[1-\left(\frac{V}{V_{0}}\right)^{1 / 3}\right]\right]
\end{aligned}
$$

where, $B_{0}$ is the isothermal bulk modulus at room temperature and ambient pressure, while $B_{0}^{\prime}$ is the partial derivative of the isothermal bulk modulus against pressure under the same conditions. This constitutive relation was shown to be universally valid for all solids under a wide range of pressure values [21]. Note that the Vinet equation of state is significantly different than the simple $P=B_{0}$ $\left(V / V_{0}-1\right)$ relation valid for isotropic solids at the small strain approximation [11]. As will be shown in the next section, the high pressures employed in the present investigation led to large volume changes justifying the use of the Vinet equation. Here, the volume change vs. pressure data were fit with this equation via the least squares method yielding values for $B_{0}$ and $B_{0}^{\prime}$.

A note of caution is in order before proceeding with the results of the deformation analysis. The large discrepancy between the bulk moduli of BMG and W (see the next section) means that the applied pressure will not be equally shared in the composite. Assuming the interface between the phases is intact for all pressure values, the higher modulus of $\mathrm{W}$ will force it to sustain higher pressure than that felt by the BMG matrix. A reasonable estimate of the pressure values for each phase can be obtained using the Eshelby model [10]. Here, both phases were assumed to remain elastic and the following values were employed for their elastic constants: Young's modulus, $E_{\mathrm{BMG}}=85 \mathrm{GPa}$, Poisson's ratio, $v_{\mathrm{BMG}}=0.38$ [3]; $E_{\mathrm{W}}=410 \mathrm{GPa}, \nu_{\mathrm{W}}=0.28$ [8]. The results of the Eshelby

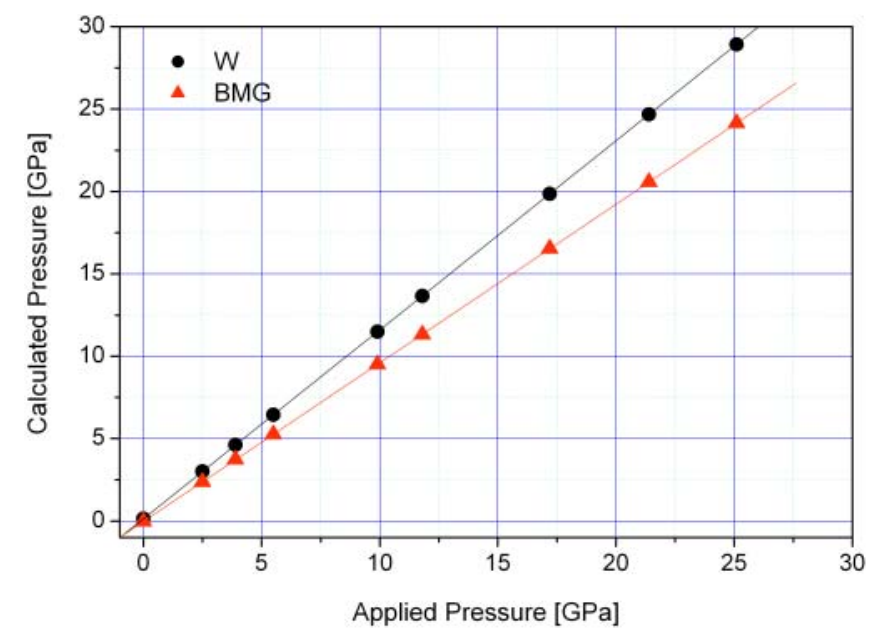

Fig. 6. Calculated pressure (via the Eshelby model) vs. applied pressure for both $\mathrm{BMG}$ and $\mathrm{W}$ in the composite.
Table 1. Bulk modulus data for BMG and W. The error values in the third column were calculated with respect to the literature data: $B_{0}=118 \mathrm{GPa}(\mathrm{BMG}), B_{0}=310 \mathrm{GPa}(\mathrm{W})$. The error bars for the $B_{0}$ values resulted from the least square fitting of Eq. (3).

\begin{tabular}{lcl}
\hline & $B_{0}(\mathrm{GPa})$ & Error $(\%)$ \\
\hline BMG in monolith (PDF) & $112( \pm 2)$ & 5 \\
BMG in monolith (XRD) & $164( \pm 11)$ & 39 \\
& & \\
BMG in composite (PDF), $P_{a}{ }^{a}$ & $90( \pm 3)$ & 24 \\
BMG in composite (PDF), $P_{c}{ }^{b}$ & $86( \pm 3)$ & 27 \\
BMG in composite (XRD), $P_{a}{ }^{a}$ & $155( \pm 8)$ & 31 \\
BMG in composite (XRD), $P_{c}{ }^{b}$ & $149( \pm 7)$ & 26 \\
W (Rietveld), $P_{a}{ }^{a}$ & $344( \pm 13)$ & 11 \\
W (Rietveld), $P_{c}{ }^{b}$ & $397( \pm 15)$ & 28 \\
\hline
\end{tabular}

a: Obtained using applied pressure data

b: Obtained using calculated pressure data

calculation in comparison with applied pressure data are shown in Fig. 6. It is clear that the two phases do not experience the same pressure values and there are significant differences between the two: e.g., at $25 \mathrm{GPa}$ applied pressure, the calculated value for BMG is $24 \mathrm{GPa}$ whereas that for $\mathrm{W}$ is $29 \mathrm{GPa}$.

\section{Results and discussion}

Table 1 summarizes the results for bulk moduli obtained from different analyses. The $B_{0}$ values calculated by fitting the data to the Vinet equation (Eq. (3)) are exhibited in comparison to the literature values. The latter moduli were calculated from the Young's modulus and Poisson's ratio values given above using the following relationship valid for elastically isotropic materials (both BMG and $\mathrm{W}$ fall under this category): $3 B_{0}=E /(1-2 v)$.

A few additional comments about the calculations are in order:

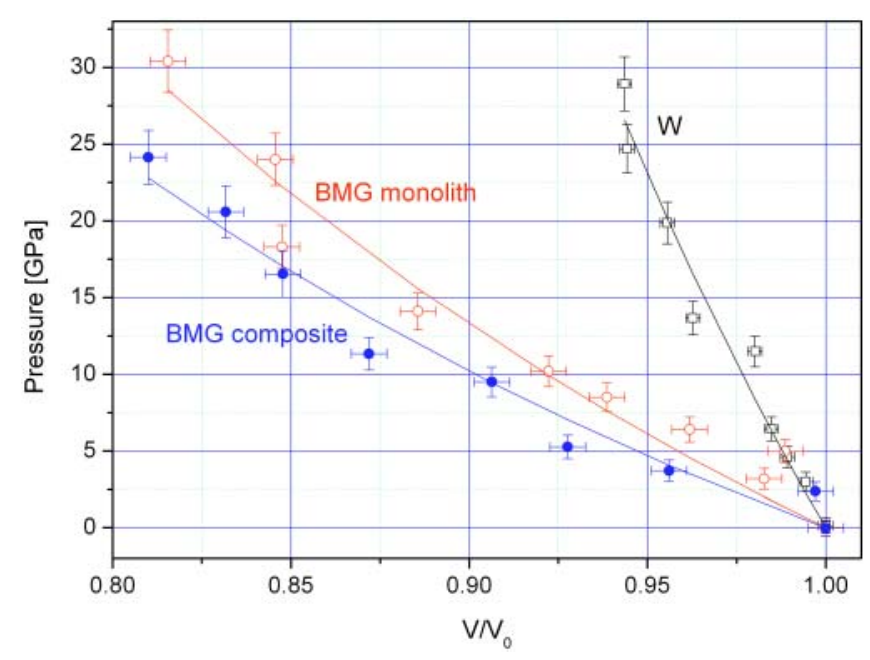

Fig. 7. Relative volume change vs. pressure for monolithic BMG and $20 \%$ W-BMG composite. For the latter, the pressure values calculated by the Eshelby model were used. For all three data sets, the Vinet function (Eq. (3)) fits are also shown. 
- The BMG volume change data were obtained either from the PDF or XRD patterns. In each case, the position of the first peak was tracked as a function of pressure and Eq. (2) was utilized.

- To observe the effect of the correction in the pressure values for each phase of the composite, results are shown from both the applied and calculated pressure data.

- The $B_{0}$ estimates are sensitive to the value of $B_{0}^{\prime}$. This is especially true for data sets with few data points in the low pressure regime [21]. As can be seen in Fig. 7, the present data set includes about 10 data points and is not as extensive as some data sets described in [21]. To assure a proper comparison of different analyses in the present study, the value of $B_{0}^{\prime}$ was fixed to 2.2 for all $\mathrm{BMG}$ fits. This was obtained from the two-parameter fitting of the monolith PDF data as it offered the best fit. Similarly, the $B_{0}^{\prime}$ for $\mathrm{W}$ was fixed to 4.9 obtained from the two-parameter fitting of the applied pressure data. Note that the $B_{0}^{\prime}$ values for either phase are within the range reported in [21] for a variety of solids. Also, the regression numbers $\left(R^{2}\right)$ for all fits ranged between 0.90 and 0.98 indicating good fits between data and the Vinet equation.

The results exhibited in Table 1 can be summarized as follows:

1. For the BMG monolith, the PDF pattern yielded a much better value for the bulk modulus (with only a $5 \%$ error) compared to the XRD data. This can be attributed to the smoother and better defined peaks in the PDF patterns.

2. The bulk moduli obtained from the BMG in the composite had larger errors (about 25-30\%). Since the $\mathrm{W}$ pattern was subtracted from the composite data before the fitting of the BMG peaks, distortions occurred in the BMG patterns and those likely led to poorer peak fits. A possible solution to this problem is simultaneous fitting (e.g., via the Rietveld method) of two separate crystallographic models for the $\mathrm{W}$ and $\mathrm{BMG}$. This work is currently in progress.

3. The bulk moduli obtained for the BMG are reasonable enough to show that one can track the position of the first BMG peak and obtain sensible estimates of elastic strain due to hydrostatic pressure.

4. The correction of the pressure values using the Eshelby model on the composite did not result in any appreciable improvement. In fact, the bulk modulus of $\mathrm{W}$ obtained this way showed larger error (28\%) compared to that from the applied pressure data (11\%). There are two possible reasons for this observation. First, the Eshelby model is strictly valid within the small strain approximation. The $\mathrm{W}$ and BMG volume changes measured at high pressure values approached 7 and $20 \%$, respectably. This is a regime where the applicability of the Eshelby model is questionable. Another possible reason for the larger error in the calculated pressure data is the size of the X-ray spot $(\sim 50 \mu \mathrm{m}$ in diameter) compared to the $\mathrm{W}$ particle size $(\sim 10 \mu \mathrm{m}$ diameter on aver- age). It is therefore probable that very few $\mathrm{W}$ particles fell within the sampling volume, or even worse, some of those might not have been fully enclosed by the BMG matrix. If the latter case were true, then W would experience the applied pressure directly.

\section{Conclusions}

A diamond anvil cell was used to study the deformation under hydrostatic pressure of a monolithic BMG alloy $\left(\mathrm{Zr}_{57} \mathrm{Nb}_{5} \mathrm{Al}_{10} \mathrm{Cu}_{15.4} \mathrm{Ni}_{12.6}\right)$ and its composite with 20 vol.\% $\mathrm{W}$ particles. Energy dispersive XRD was employed to track the structural evolution of both phases in situ. No pressure-induced phase change was detected in either BMG or W. It was also shown that the shift of the first peak in the BMG diffraction pattern can be used to obtain a reasonable estimate of its elastic strain. The PDF pattern of the material appeared to yield better strain accuracy via this approach.

While these preliminary results are encouraging, there are additional studies underway to obtain deeper insight into the deformation of BMG. First, molecular dynamics simulations [17] are being performed to not only study the atomistic structure of BMG under stress/pressure but to also quantitatively relate the evolution of its PDF pattern to the average elastic strain in the material. This way, a direct comparison can be made with the experimental PDF data. One of the challenges in this study is to understand how uniaxial loading, common in most applications, affects the structure of BMG. In a second study, neutron diffraction is being used to collect PDF patterns of BMG under uniaxial compression [22]. A shortcoming of the XRD study presented here is that it yields a limited range of $Q$ space. It is well known that cutoff at low $Q$ decreases the real space resolution of PDF and causes termination ripples [15]. Neutron diffraction as well as high energy synchrotron XRD in angle dispersive mode solve this problem since both techniques offer access to a much larger $Q$ range.

In sum, the unique properties of BMGs (low modulus, high strength) position them as ideal candidates to employ their amorphous pattern peaks for strain measurement. However, strain resolution obtained from these amorphous alloys will always be inferior to that attained from diffraction patterns of crystalline materials. Nevertheless, the BMG strain data can complement the data from the crystalline reinforcements in BMG composites.

Acknowledgments. This study was supported by the National Science Foundation (CAREER Award to E.Ü.: DMR-9985264) and the Caltech Center for Structural Amorphous Metals (Army Research Office grant no. DAAD19-01-0525). The work at the National Synchrotron Light Source (NSLS) was funded by the U.S. Department of Energy under Contract No. DE-AC02-76CH00016.

\section{References}

[1] Peker, A.; Johnson, W. L.: A highly processable metallic-glass - $\mathrm{Zr}_{41.2} \mathrm{Ti}_{13.8} \mathrm{Cu}_{12.5} \mathrm{Ni}_{10.0} \mathrm{Be}_{22.5}$. Appl. Phys. Lett. 63 (1993) $2342-2344$. 
[2] Choi-Yim, H.; Johnson, W. L.: Bulk metallic glass matrix composites, Appl. Phys. Lett. 71 (1997) 3808-3810.

[3] Choi-Yim, H.; Busch, R.; Koster, U.; Johnson, W. L.: Synthesis and characterization of particulate reinforced $\mathrm{Zr}_{57} \mathrm{Nb}_{5} \mathrm{Al}_{10} \mathrm{Cu}_{15.4} \mathrm{Ni}_{12.6}$ bulk metallic glass composites. Acta Materialia 47 (1999) 2455-2462.

[4] Conner, R. D.; Choi-Yim, H.; Johnson, W. L.: Mechanical properties of $\mathrm{Zr}_{57} \mathrm{Nb}_{5} \mathrm{Al}_{10} \mathrm{Cu}_{15.4} \mathrm{Ni}_{12.6}$ metallic glass matrix particulate composites. J. Mater. Res. 14 (1999) 3292-3297.

[5] Balch, D. K.; Üstündag, E.; Dunand, D. C.: Elasto-plastic load transfer in bulk metallic glass composites containing ductile particles. Metallurgical Materials Transactions 34A (2003) 1787-1797.

[6] Balch, D. K.; Üstündag, E.; Dunand, D. C.: Diffraction strain measurements in a partially crystallized bulk metallic glass composite containing ductile particles. J. Non-Crystall. Solids 317 (2003) 176-180.

[7] Dragoi, D.; Üstündag, E.; Clausen, B.; Bourke, M. A. M.: Investigation of thermal residual stresses in tungsten-fiber/bulk metallic glass matrix composites. Scripta Materialia 45 (2001) $245-252$.

[8] Clausen, B.; Lee, S. Y.; Üstündag, E.; Aydiner, C. C.; Conner, R. D.; Bourke, M. A. M.: Compressive yielding of tungsten fiber reinforced bulk metallic glass composites. Scripta Materialia 49 (2003) 123-128.

[9] Suzuki, Y.; Haimovich, J.; Egami, T.: Bond-orientational anisotropy in metallic glasses observed by X-ray diffraction. Phys. Rev. B35 (1987) 2162-2168.

[10] Eshelby, J. D.: The determination of the elastic field of an ellipsoidal inclusion, and related problems. Proceedings of the Royal Society of London Series A - Mathematical and Physical Sciences 241 (1957) 376-396.
[11] Noyan, I. C.; Cohen, J. B.: Residual Stress: Measurement by Diffraction and Interpretation. Springer Verlag, New York, 1987.

[12] Sterer, E.; Pasternak, M. P.; Taylor, R. D.: A multipurpose miniature diamond anvil cell. Rev. Scientific Instruments 61 (1990) $1117-1119$.

[13] Jayaraman, A.: Diamond anvil cell and high-pressure physical investigations. Rev. Modern Physics 55 (1983) 65-108.

[14] He, D. W.; Zhao, Q.; Wang, W. H.; Che, R. Z.; Liu, J.; Luo, X. J.; Wang, W. K.: Pressure-induced crystallization in a bulk amorphous Zr-based alloy. J. Non-Crystalline Solids 297 (2002) 8490.

[15] Proffen, Th.; Billinge, S. J. L.; Egami, T.; Louca, D.: Structural analysis of complex materials using the atomic pair distribution function - a practical guide. Z. Kristallogr. 218 (2003) 132143.

[16] Petkov, V.: RAD: A program for analysis of X-ray-diffraction data from amorphous materials for personal computers. J. Appl. Crystallogr. 22 (1989) 387-389.

[17] Cagin, T.; Üstündag, E.: Molecular dynamics simulation of bulk metallic glass deformation. In preparation (2004).

[18] Meade, C.; Hemley, R. J.; Mao, H. K.: High-pressure X-ray diffraction of $\mathrm{SiO}_{2}$ glass. Phys. Rev. Lett. 69 (1992) 1387-1390.

[19] Rietveld, H. M.: A profile refinement method for nuclear and magnetic structures. J. Appl. Crystallogr. 2 (1969) 65-71.

[20] Wiles, D. B.; Young, R. A.: A new computer-program for Rietveld analysis of X-ray-powder diffraction patterns. J. Appl. Crystallogr. 14 (1981) 149-151.

[21] Vinet, P.; Ferrante, J.; Rose, J. H.; Smith, J. R.: Compressibility of solids. J. Geophys. Res. Solid Earth Planets 92 (1987) 93199325.

[22] Lee, S. Y.; Proffen, Th.; Clausen, B.; Üstündag, E.: Neutron diffraction study of bulk metallic glass structure under compression. In preparation (2004). 